\title{
Une dogmatique de l'espace. Les lieux de la loi en Chine impériale
}

Jérôme Bourgon

\section{OpenEdition}

1 Journals

Édition électronique

URL : http://journals.openedition.org/extremeorient/597

DOI : 10.4000/extremeorient.597

ISBN : 978-2-84292-558-1

ISSN : 2108-7105

Éditeur

Presses universitaires de Vincennes

Édition imprimée

Date de publication : 21 novembre 2016

Pagination : 5-12

ISBN : 978-2-84292-556-7

ISSN : 0754-5010

\section{Référence électronique}

Jérôme Bourgon, «Une dogmatique de l'espace. Les lieux de la loi en Chine impériale », Extrême-Orient Extrême-Occident [En ligne], 40 | 2016, mis en ligne le 21 novembre 2016, consulté le 24 septembre 2020. URL : http://journals.openedition.org/extremeorient/597 ; DOI : https://doi.org/10.4000/ extremeorient.597 


\title{
Une dogmatique de l'espace. Les lieux de la loi en Chine impériale
}

\author{
Jérome Bourgon
}

« Territorium est universitas agrorum infra fines cujusque civitatis, quod ab eo dictum quidam aiunt, quod magistratus ejus loci intra eos fines terrendi, id est summovendi jus habent. »

[Un territoire est l'ensemble des terres situées dans les limites d'une cité, dont certains disent qu'il est ainsi nommé parce que le magistrat de ce lieu a dans ces limites le droit de terroriser, c'est-à-dire d'exclure.] Pomponius, Digeste, 50, 16, 239, 8 .

Qu'on nous pardonne de commencer par un quizz sans rapport avec la Chine : se souvient-on de la teneur de l'article Premier du Code civil de 1804, dit «Code Napoléon », ce monument du droit moderne auquel se rattachent encore de nombreux systèmes juridiques nationaux du monde actuel ? Il ne ressemble à rien de ce que nos conceptions courantes du droit laisseraient attendre. Le voici :

\section{Article Premier}

Les lois sont exécutoires dans tout le territoire français en vertu de la promulgation qui en est faite par le Premier Consul.

Elles seront exécutées dans chaque partie de la République, du moment où la promulgation pourra être connue.

La promulgation faite par le Premier Consul sera réputée connue dans le département où siègera le Gouvernement, un jour après celui de la promulgation ; et dans chacun des autres départements, après l'expiration du même délai, augmenté d'autant de jours qu'il y aura dix myriamètres (environ vingt lieues anciennes) entre la ville où la promulgation en aura été faite, et le chef-lieu de chaque département ${ }^{1}$.

1. Code civil des Français, Titre préliminaire, 1804. Édition originale téléchargeable sur [http://www.legilux.public.lu/leg/a/archives/1804/0005/a005.pdf]. 
Pas de solennelle proclamation de droits individuels ou de principes éternels, juste la mise en place d'un dispositif de diffusion à partir d'un centre de pouvoir. Pour n'être pas qu'un « art simple et tout d'exécution », comme Napoléon le disait de la guerre, le droit n'en a pas moins sa logistique. Durant l'essentiel des temps historiques, la loi s'est déplacée d'un relais de poste à l'autre à la vitesse d'un cheval au galop : quatre-vingt à cent kilomètres par jour en moyenne, sous Napoléon comme sous ses contemporains Qianlong et Jiaqing. Le législateur avisé sait que l'expression de sa volonté doit prendre le temps de la transmission, et compter avec les divers obstacles que la matérialité de l'espace lui oppose.

C'est dans cet esprit que nous avons entrepris d'étudier l'empire chinois comme espace modulé par le droit. «Régir l'espace chinois : la structuration du territoire de la Chine impériale par un système juridique hiérarchisé », tel était le titre du projet financé par l'Agence nationale de la recherche (ANR) qui s'est conclu par le colloque sur « Les lieux de la loi » dont les communications ont servi de point de départ à la constitution de ce numéro d'Extrême-Orient, Extrême-Occident ${ }^{2}$. L'empire des Qing se présente en effet comme un agencement de territoires obéissant à des normes originellement très diverses - code pénal et ordonnances réglementaires pour la Chine des dix-huit provinces héritées des Ming, lois et coutumes spécifiques aux régions mongoles, tibétaines, ouighoures, ou à d'autres moins étendues comme celle des populations Miao, etc. Comment cet agrégat inconstitué de peuples désunis s'est-il trouvé graduellement intégré, sinon unifié, en un même système juridique ? Certes, l'empire chinois n'a jamais atteint le degré de centralisation et d'homogénéité de la France post-révolutionnaire. Son système juridique est resté composite, ou " pluraliste » comme l'était l'administration dans son ensemble. La tendance était pourtant bien à l'unification sous une même loi, comme le montrent les divers articles réunis ici. Tout leur mérite et, espéronsle, leur intérêt, sont d'exposer savamment les modes d'opération visant à convertir des espaces conquis en territoires soumis, au prix des nombreuses variations imposées par les diversités locales.

Il nous a paru commode de distinguer les modes d'opération tendant à «Pénaliser l'espace » et à « Réglementer l'espace ».

Les articles rassemblés sous le premier thème paraissent autant d'illustrations de l'extrait de Pomponius cité en exergue. Selon une étymologie douteuse mais bien établie sous l'autorité des commentateurs du code Justinien, base romaine des droits européens, le mot territoire désigne un lieu dans les limites

2. Les objectifs, les membres et les réalisations de ce projet sont consultables sur [http:// lsc.chineselegalculture.org]. 
duquel le magistrat a tout droit de «terroriser $»^{3}$. C'est bien le genre de pouvoir dont il s'agit dans l'article qu'Eric Schluessel consacre aux « exécutions sur le champ » en territoire ouighour, qui dérogeaient aux procédures légales de vérification et de révision des peines capitales. Le régime d'exception d'un pouvoir colonial s'exerçant en territoire « indigène » musulman au nom d'une mission civilisatrice mettait le rite, instrument présumé de la civilisation et mobile très noble de la terreur, au-dessus des lois. C'est bien ce même arbitraire qui caractérisait la « justice militarisée » (militarized adjudication) étudiée par John Gregory. Son symbole est la bannière portant les caractères gong qing wang ming (dans l'attente respectueuse d'un ordre souverain) qui flottait sur les lieux d'exécutions sommaires, en hommage ironique et sinistre à la procédure exigeant que toute peine capitale fût expressément autorisée par rescrit impérial. Gregory montre que le recours à cette sorte de justice expéditive était déterminé par la situation géographique du condamné, et avait toute chance de prévaloir dans les «zones de combat » et les régions frontières.

C'est un autre genre de pénalisation de l'espace que décrit Frédéric Constant, comme pour éclairer l'étymologie de Pomponius sous son autre face : un territoire est un lieu où le magistrat peut terroriser, c'est-à-dire exclure. De fait, les diverses formes d'exil et de déportation pratiquées depuis l'Antiquité apparurent d'abord comme substitut de la peine capitale, ce qui permettait de joindre les attraits de la clémence aux avantages de l'exclusion sociale et aux profits du travail forcé. Rien là de bien original, mais ce qui distingue la Chine, c'est d'avoir systématisé cette utilisation pénale de l'espace en la liant étroitement à l'échelle de peines légales qui était au fondement de son système juridique codifié. L'exil, qui formait le quatrième étage de l'ensemble des Cinq peines légales (Wuxing), fut ainsi subdivisé en une série de gradations fondées sur l'éloignement et la nocivité des lieux. Constant montre comment les Ming et, surtout, les Qing s'astreignirent à rendre effective sur le terrain cette gamme d'aggravations au moyen de tableaux complexes calculant les distances de la préfecture de départ à la préfecture d'arrivée, et tenant compte de la qualité des lieux « insalubres », «frontaliers », ou habités par des populations farouches. Les dimensions et les qualités du territoire deviennent alors autant de moyens de moduler la peine d'exil et de " punir par l'espace».

Il est un autre aspect sur lequel cet article très riche attire notre attention : c'est l'infrastructure dogmatique qui fonde l'ensemble de ces opérations complexes. Ce qui passerait aisément pour une succession de mesures

3. La citation de Pomponius n'est que la plus explicite parmi d'autres fondées sur cette étymologie, qui semble confirmée par des dérivés comme l'adjectif territor, « qui répand la terreur (épithète de Jupiter) ». Voir Gaffiot 1934 : 1561. 
pratiques visant à adapter un vieux système aux nécessités du temps apparaît à meilleur examen comme le déploiement d'une conception de l'espace qui embrasse l'ensemble des institutions. C'est ainsi que la gradation des exils répond au schéma dit des $W u f u$, soit Cinq phases de dégradation progressive au fur et à mesure qu'on s'éloigne du centre civilisé vers les marges barbares. Que ces cinq fu spatiaux soient désignés du même caractère, renvoyant au même concept, que les cinq $f u$ qui désignent les degrés de deuil structurant l'espace familial est un de ces raccourcis fulgurants qui font l'efficacité des procédés dogmatiques ${ }^{4}$. Nous avons affaire au développement spatial d'un ensemble dogmatique coulant dans un même bronze l'échelle des peines, la gradation des lieux d'exil, la hiérarchie des rangs familiaux, et l'empire comme espace de civilisation rayonnant à partir d'un centre. C'est cette structure d'ensemble que les savants philologues ou les experts juristes savaient mobiliser le moment venu, chacun dans son domaine et selon ses nécessités propres. Ce qui peut paraître un exercice de style ou un jeu de fictions est une clé de la pérennité des institutions impériales.

Le cas des populations Miao, étudié par Zhang Ning, montre le lien intime entre pénalisation du crime et intégration au territoire. Tant que le territoire des Miao fut jugé difficilement assimilable car peuplé de Barbares « crus » selon la typologie chinoise, ceux-ci purent conserver leurs mœurs comme autant de « règles coutumières » appliquées par des chefs locaux reconnus par les Qing. Sitôt décidée l'intégration à l'empire, des lois furent codifiées pour criminaliser lesdites « règles coutumières », à savoir les vendettas entre lignages ou entre villages, les enlèvements contre rançon, et le trafic de personnes, principalement de femmes et d'enfants en direction du Sichuan. Alors qu'avaient été admises les pratiques consistant à racheter les homicides commis lors des vendettas par des compensations en bétail ou en numéraire, les Miao furent graduellement soumis aux procédures et aux peines réprimant le meurtre. La vieille pratique de l'enlèvement contre rançon fut poursuivie en liaison avec les opérations ciblant le trafic d'êtres humains par des bandes de criminels Han. C'est donc d'un même mouvement que le « territoire des Miao » fut intégré à l'empire et que certaines de leurs pratiques entrèrent dans le code des Qing comme autant de crimes à punir.

Le cas des Miao offre une bonne transition vers notre second thème : « Réglementer l'espace », ou, dans sa formulation anglaise, Legalizing Space.

4. Dogmatique s'entend ici au sens donné par Pierre Legendre, qui peut se résumer à « une architecture de significations ordonnées pour renvoyer aux origines dès qu'est en question la légitimité du pouvoir ». Voir Legendre 2009 : 51. 
Il s'agit de la mise en forme du territoire par une législation différenciée, supposément adaptée aux nécessités du lieu.

L'article de Wang Zhiqiang donne un aperçu général de la question. Les « lois spéciales à caractère régional » qu'il a étudiées ne sont pas des règles locales, pas même des « règles coutumières » qui auraient été reconnues par les Qing puis intégrées à leurs codes. C'étaient des lois édictées par l'autorité centrale en considération des particularités d'un endroit donné, particularités qui étaient souvent signalées par, ou négociées avec, la bureaucratie territoriale en charge de ces régions. Décrétées par les autorités centrales aux fins de défense de la capitale, de contrôle du territoire et de protection des frontières, ces lois spéciales furent intégrées au code des Qing sous forme d'articles additionnels. C'étaient autant d'exceptions locales à la loi générale, qui assouplissaient ou allégeaient parfois, mais le plus souvent aggravaient les peines prévues pour certains types de crimes jugés sensibles dans la région concernée. Il s'agit, pour simplifier, d'un coefficient d'aggravation des peines dans les zones stratégiques, qui permet de cartographier assez précisément la manière dont les Qing concevaient le contrôle de leur territoire.

La réglementation de l'espace visait aussi à organiser les tâches ordinaires de l'administration en fonction des contraintes géographiques. C'est ainsi que les règles extrêmement pointilleuses régissant les autopsies durent être modifiées et, en fin de compte, assouplies, dans des régions montagneuses et chaudes de la Chine du Sud, où les cadavres risquaient de se décomposer avant que le magistrat flanqué de son agent légiste parvienne sur les lieux du crime. Xie Xin-zhe analyse tout un réseau de mesures complémentaires visant à concilier trois données : 1 . les précautions légales entourant l'autopsie, source de possibles erreurs judiciaires, exigeant qu'elle soit faite par l'autorité compétente selon une procédure et dans des délais strictement définis ; 2 . les contraintes de l'espace (éloignement, escarpement, climat tropical, etc.) ; 3. la rareté des magistrats et des experts qualifiés sur le terrain. Ces contraintes trouvent leur moyen terme dans la délégation du pouvoir d'autopsier à des personnels subalternes sous certaines conditions définies par une série d'articles additionnels dans le code des Qing. Autant de "lois spéciales à caractère régional », qui sont d'ailleurs brièvement mentionnées dans l'article de Wang Zhiqiang.

C'est un autre type de difficultés que posait l'Amdo, région de peuplement majoritairement tibétain dont Max Oidtmann nous montre comment elle fut graduellement intégrée à la province chinoise du Gansu. L'absorption se fit par la voie légale, non par la criminalisation des pratiques comme dans le cas des Miao, mais par le développement d'une jurisprudence de cas mixtes jugés par les cours de justice des Qing. Seule l'activité judiciaire, consistant 
en un ensemble cohérent de « pratiques judiciaires » (jurispractices), pouvait répondre au défi d'une pluralité qui était à la fois ethnique (Tibétains, Mongols, Han, etc.), religieuse (les bouddhistes tibétains de différentes obédiences se disputant temples et bénéfices), et même juridique, puisque la région restait encore régie par des vestiges de législations mongoles. Une telle marqueterie exigeait une certaine dextérité dans le traitement des cas fan (indigènes), que les juges des Qing entreprirent même de consolider en «lois pénales fan » (fan statutes/fan lii), créant ainsi une sorte de zone juridique tampon où l'autonomie des autorités religieuses était garantie dans la mesure où celles-ci reconnaissaient la légitimité des juridictions mises en place par les Qing pour régler leurs conflits.

C'est la logistique des fournitures aux armées et la répartition des armes de guerre qu'Ulrich Theobald étudie dans un article basé sur des recueils de règlements jusqu'ici négligés par la recherche. L'enjeu est ici de dégager de la simple routine réglementaire ceux des « règlements » ou « précédents », ou « exemples » qui représentaient une réelle amélioration en terme d'adaptation du système aux nécessités des temps et des lieux. S'agissant de la logistique militaire, il fallait à la fois assurer une présence assez constante sur l'ensemble du territoire, et rester prêt à parer à toute éventualité en concentrant des forces dans les endroits menacés. L'effort de standardisation des équipements et des fonctionnements se heurte ici à la nature composite des armées des Qing - les bataillons verts des Han étaient par leur mode d'organisation et de commandement profondément différents des Bannières, qui différaient elles-mêmes selon qu'elles étaient issues d'unités mandchoues ou mongoles ainsi qu'à une gestion très décentralisée des forces armées. L'innovation réglementaire joua finalement en faveur de l'autonomie locale plutôt que du progrès technologique d'ensemble, avec les résultats que l'on sait lors des guerres de l'opium.

Qu'un empire aussi vaste ait dû affronter des problèmes si divers n'est pas surprenant. Ce qui l'est en revanche, c'est qu'il ait pu les traiter, sinon les résoudre, avec une assez remarquable constance et avec un certain succès durant deux siècles, ou même deux fois deux siècles si l'on inclut dans le raisonnement la dynastie des Ming. En prenant davantage de recul encore, le plus étonnant est que les mêmes institutions aient pu résister et évoluer sur environ six siècles, de l'avènement des Ming (1368) aux grandes réformes du début du $\mathrm{XX}^{\mathrm{e}}$ siècle, par-delà la transition inter-dynastique particulièrement brutale du milieu du XVII ${ }^{\mathrm{e}}$ siècle. C'est à une telle réflexion de longue durée que nous invite le « regard extérieur» de Jean-Louis Halpérin. À la différence d'autres civilisations tout aussi respectables, mais à l'instar de l'empire romain, l'empire chinois était fondé sur un système juridique qui connut une évolution 
millénaire. Il faut entendre par là non un système de normes fondamentales, si sophistiquées ou sacrées fussent-elles, mais le fait que ces normes primaires aient été elles-mêmes soumises à trois genres de "règles secondaires », ou «procédurales ». La première détermine quelles sont les règles impératives (en promulguant un code de lois, par exemple), la deuxième indique qui a le pouvoir de les changer et comment (en ajoutant des articles additionnels dans le code), la troisième désigne qui a le pouvoir de les appliquer dans des jugements (en nommant pour cela des magistrats). Seul un système comportant ces deux étages bien différenciés et savamment articulés peut être qualifié de " juridique », nous indique Jean-Louis Halperin, s'inspirant des thèses de Herbert Hart. Le dynamisme propre à ce genre de systèmes leur permet non seulement de répondre par des mesures appropriées à la grande variété de situations imprévues propre à tout vaste empire, mais aussi de conserver à l'ensemble une cohérence doctrinale indispensable à sa pérennisation, ou à sa reconstitution en cas d'effondrement. Une telle cohérence inspire aux élites dirigeantes le sentiment qu'elles œuvrent au triomphe de principes de justice universels, dont la diffusion exige de normaliser un espace toujours grandissant autour du centre civilisateur.

Ce sont donc quelques aspects spatiaux de cet ensemble dogmatique que nous présentons ici, et nous en remercions la revue Extrême-Orient, ExtrêmeOccident.

\section{BIBLIOGRAPHIE}

Code civil des Français. Paris : Imprimerie de la République, 1804.

GAFFIOT, Félix (1934). Dictionnaire latin-français. Paris : Hachette.

LEGENDRE, Pierre (2009). Leçon IX. L'autre Bible de l'Occident : le Monument romanocanonique. Étude sur l'architecture dogmatique des sociétés. Paris : Fayard.

\section{GLOSSAIRE}

fan 番

Gansu 甘肅

gong qing wang ming 恭請王命

Han 漢

Jiaqing 嘉慶

$l i$ 例 
Jérôme Bourgon

Miao 苗

Miaoli 苗例

Ming 明

Qianlong 乾隆

Qing 清

tiaoli 條例

Wufu 五服

Wuxing 五刑

$z e l i$ 則例 